\title{
SURGERY ON POINCARÉ AND NORMAL SPACES ${ }^{1}$
}

\author{
BY FRANK QUINN \\ Communicated by M. F. Atiyah, October 4, 1971
}

1. The object of this note is to announce a theory of surgery on Poincaré and normal spaces, with applications to Poincaré geometry and manifolds.

The present point of view on Poincare surgery was outlined by $\mathrm{W}$. Browder in the spring of 1969 , and is purely homotopy-theoretic. The main missing ingredient in the program was Lemma 1.5, and the (considerable) machinery required for its application. Other approaches to Poincaré geometry have been made by N. Levitt [3], [4] using engulfing and manifold surgery, and by L. Jones [2] using patch structures (see 2.4 below).

Results in this area have also been obtained recently by W. Browder, A. S. Mischenko, and C. Morlet.

By a Poincaré space we mean one which satisfies duality with local coefficients (Wall [8]). Duality then gives a chain equivalence of the chains and cochains, which has a Whitehead torsion. This is the torsion of the space. The surgery groups $L^{s}, L^{h}$, etc. are encountered according to restrictions put on the torsion of the spaces considered. We neglect further mention of the torsion.

If $X$ is Poincare, one constructs the Spivak normal fibration $v_{X}^{k}$ with Thom class $U$, which is distinguished by a map $\rho: S^{n+k} \rightarrow T v_{X}$ such that $\rho_{*}\left[S^{n+k}\right] \cap U=[X]$. An abstraction is useful.

1.1. Definition. A normal space is an $X$ with a $S^{k-1}$ fibration $\xi_{X}$ and a map $\rho_{X}: S^{n+k} \rightarrow T \xi_{X}$. A normal map of normal spaces is a map $f: X \rightarrow Y$ with $\xi_{X} \simeq f^{*} \xi_{Y}$, and $\rho_{Y}=T f^{*} \circ \rho_{X}$.

The fundamental class of $X$ is $\rho_{X}\left[S^{n+k}\right] \cap U$, and has dimension $n$. A normal map is automatically of degree 1 . Normal pairs $(X, Y)$ have a map $\rho: S^{n+k} \rightarrow T \xi / T(\xi \mid Y)$.

The usefulness of normal spaces is twofold. First transversality holds for spherical fibrations in the normal category. Thus if pairs are used to define the normal bordism groups $\Omega_{*}^{N}(X)$, we get $\Omega_{*}^{N}(X) \simeq H_{*}(X ; M S G)$. Secondly the mapping cylinder of a normal map is a normal pair. Therefore knowledge of the obstructions to improving a normal space to be Poincaré gives, by the first remark obstructions to Poincaré transversality, and Steenrod

AMS 1970 subject classifications. Primary 57B10, 57C60; Secondary 55D05, 55B20.

Key words and phrases. Surgery, Poincaré space, normal space, cofibrations, Poincaré bordism.

${ }^{1}$ This work was partially supported by National Science Foundation grant GP 29701 at the Courant Institute of New York University and GP 7952X3. 
representability, and by the second remark, obstructions to Poincaré surgery.

Our main theorem is the following.

1.2. THEOREM. If $\left(X ; Y_{0}, Y_{1}\right)$ is a normal triad of dimension $n$, with $\left(Y_{0}, Y_{0} \cap Y_{1}\right)$ Poincaré (and a $P L$ manifold if $\left.n=3,4\right)$, and $\pi_{1} Y_{1} \simeq \pi, X$, then there is a normal map $\left(W ; Y_{0}, Z\right) \rightarrow\left(X ; Y_{0}, Y_{1}\right)$ with $\left(W ; Y_{0}, Z\right)$ Poincaré.

This is used to construct a normal bordism of an arbitrary normal space to the mapping cylinder of a smooth surgery map, showing the obstructions are the same as the smooth case.

1.3. THEOREM. If $\left(X ; Y_{0}, Y_{1}\right)$ is normal of dimension $n \geqq 5\left(4\right.$ if $\left.Y_{1}=\varnothing\right)$, $\left(Y_{0}, Y_{0} \cap Y_{1}\right)$ Poincaré, then there is an obstruction $\sigma(X) \in L_{n-1}\left(\pi_{1} Y_{1} \rightarrow \pi_{1} X\right)$ which vanishes iff $\left(X ; Y_{0}, Y_{1}\right)$ is normally bordant, keeping $\pi_{1}$ and $Y_{0}$ fixed, to a Poincaré triad (or if $X$ is a normal Poincaré image as in 1.2).

1.4. Corollary. The obstruction to a normal map of Poincaré spaces being normally cobordant to a homotopy equivalence is the same as the manifold case.

Sketch OF Proof of 1.2. Dimensions less than 5 use obstruction theory and PL transversality. For $n \geqq 5$ we indicate only the absolute case below the middle dimension.

Let $(X, \xi, \rho)$ be normal, then kernels $K_{*}(X)$ are defined so that

$$
\rightarrow K_{j}(X) \rightarrow H^{n-j}(X) \stackrel{\cap[X]}{\longrightarrow} H_{j}(X) \rightarrow K_{j-1}(X) \rightarrow
$$

is exact. We say $X$ is $j$-Poincaré if $K_{m}(X)=0, m \leqq j . K_{m}(X) \simeq K^{n-m-1}(X)$, so if $X$ is $[(n-1) / 2]$ Poincaré then it is Poincaré. The construction proceeds by induction on $j$. If $K_{j}(X)$ is the first nonvanishing kernel, a Hurewicz type theorem gives geometric representatives for elements of $K_{j}$. The images in $H^{n-j}(X)$ are cohomotopy elements $\theta: X \rightarrow S^{n-j}$.

The argument is completed by extending $\theta$ backwards as a cofibration: $S^{n-j-1} \rightarrow Y \rightarrow X \stackrel{\theta}{\rightarrow} S^{n-j}$. Exact sequences reveal $Y$ is essentially $X$ with $\theta$ killed in $K_{j}(X)$. Killing generators of $K_{j}(X)$ completes the induction.

To obtain such cofibrations we use a lemma (a more complicated version is required for $\pi_{1} X \neq 1$ and in the middle dimension).

1.5. LEMMA. If $X$ is a 1-connected $n$-complex, $\theta: X \rightarrow S^{m}$ a map with $m \geqq(n+1) / 2$, then there is a cofibration sequence $S^{m-1} \rightarrow Y \rightarrow X \stackrel{\theta}{\rightarrow} S^{m}$ if and only if $\left.\theta \wedge\right|_{X}: X \rightarrow S^{m} \wedge X$ is stability nullhomotopic.

Since $X \rightarrow S^{m} \wedge X$ induces $\bigcap[\theta]: H_{*}(X) \rightarrow \tilde{H}_{*+m}(X)$, the homotopy hypothesis is a very strong way of saying that the product of $[\theta]$ with any homology class other than its dual is zero. This indicates no higher dimensional cells are attached on top of the one we want to remove. 
To verify such homotopy hypotheses for the $\theta$ which arise in the surgery construction requires considerable preparation. A stable geometric theory of Poincaré spaces involving equivariant Spanier-Whitehead duality (of the universal cover when $\pi_{1} \neq 1$ ) and Thom spaces was developed for this. This theory seems quite useful, and has as a consequence a remark which answers a question of Wall.

1.6. REMARK. If $F \rightarrow E \rightarrow B$ is a fibration with $F, E$, and $B$ dominated by finite complexes, then $E$ satisfies Poincare duality if and only if both $F$ and $B$ do.

2. Local applications. By local we mean results concerning a particular space, rather than for example a bordism class.

2.1. Definition. If $X$ is Poincaré, $f: X \rightarrow B \cup{ }_{\xi} C$ a map where $\xi$ is an $S^{k-1}$ fibration over $C$, then $f$ is Poincaré transversal to $\xi$ if $X=\left(f^{-1} B\right.$, $\left.f^{-1} \xi\right) \cup\left(f^{-1} C, f^{-1} \xi\right)$ is a decomposition into Poincaré pairs, and $f^{-1} \xi \rightarrow f^{-1} C$ is a spherical fibration isomorphic to the pullback of $\xi$.

There is clearly a relative version, as well as a definition for normal spaces. Transversality works for normal spaces by an easy construction, so the Poincaré problem is a matter of improving normal spaces.

Suppose $X \rightarrow B \cup \xi C$ is a map where $\xi$ is a spherical fibration over $C$. Define $\pi$ as the fundamental group of the homotopy pullback

$$
\begin{aligned}
& Z \rightarrow C \\
& \downarrow \\
& X \rightarrow B \cup \cup_{\xi} C .
\end{aligned}
$$

The normal transverse inverse image of $C$ maps to $Z$, hence gives a surgery obstruction in $L_{n-k-1}(\pi)$.

2.2. (Transversality.) If $n \geqq 5, k \geqq 3$ then $\sigma\left(f^{-1}(C)\right) \in L_{n-k-1}(\pi)$ is the obstruction to making $f$ Poincaré transversal to $\xi$. If $k \leqq 2$ then the obstruction is in the group $L S_{n-k-1}(\Phi)$ defined by Wall [7, p. 126].

It is easily seen that the obstruction vanishes iff $X$ is the normal image of a Poincare space transversal to $\xi$. A sort of surgery is done on the second space preserving transversality until it is homotopy equivalent to $X$, completing the proof. This last step also applies to prove a Haefliger type embedding theorem.

2.3. (Embedding.) If $f: X^{m} \rightarrow Y^{n}$ is a map of Poincaré spaces, $n-m \geqq 3$, and $f$ is $m-[(n-1) / 2]$ connected, then $f$ is homotopic to a Poincaré embedding of $X$ in $Y$.

A little bundle theory and manifold surgery gives the usual smooth and PL theorems from this one. This result has as precursor the embedding theorems of Levitt [3], and can be used to recover the simply-connected version of 1.4 . 
The codimension 1 part of 2.2 applies to show Poincaré spaces of dimension $\geqq 5$ have smooth 2 -skeletons, a fact announced by Wall [6] and also proved by Levitt. Patch structures are an elaboration of this idea introduced by Jones [2]. They seem to be the natural replacements for handlebody structures and triangulations in the Poincaré category. A patch structure on $X$ is a homotopy equivalence of $X$ with an object formed by glueing together $n$ manifold $n$-ads via homotopy equivalences of their faces. Moreover, each face is required to have the same fundamental group as $X$.

Patch structures on normal spaces are the same, except the glueing is via degree 1 normal maps.

2.4 (Patch structures.) A Poincaré space has a smooth patch structure with no face of dimension less than 4 iff it has a cover of open sets, no $(n-3)$ of which intersect, and over each of which the normal bundle reduces to a smooth bundle. Thus a 1-connected $X$, or $X \times C P^{2}$ has a smooth patch structure.

A version of this is proved in [2] using intricate arguments on patch spaces.

3. Global applications. These are the applications to bordism and homology theories.

Let $\Omega_{n}^{P}(X)$ denote the bordism group of maps of Poincaré spaces dimension $n$ to $X$. Similarly $\Omega_{n}^{N}(X)$ is the bordism group of normal spaces, which is also $H_{n}(X ; M S G)$ as remarked above.

3.1. TheOREM. For $n \geqq 4$, the sequence

$$
\begin{gathered}
\cdots \rightarrow L_{n}\left(\pi_{1} X\right) \stackrel{\partial_{P}}{\rightarrow} \Omega_{n}^{P}(X) \stackrel{\text { incl }}{\rightarrow} \Omega_{n}^{N}(X) \rightarrow L_{n-1}\left(\pi_{1} X\right) \rightarrow \cdots \\
H_{n}(X ; M S G)
\end{gathered}
$$

is exact.

Using 1.3, $L_{n}(\pi)$ can be defined as bordism classes of normal spaces with Poincaré boundary, together with a homomorphism of $\pi_{1}$ to $\pi$. $\partial_{P}$ takes such an object to its Poincaré boundary and exactness is immediate. For $X$ a point this sequence is essentially due to Levitt [4].

Next a definition which throws some light on the structure of this sequence. Let $P$ be a normal space (with Poincaré boundary) of dimension 5 and obstruction $\sigma(P)=1 \in L_{4}(0) \simeq Z$.

3.2. Definition. If $X^{n}$ is Poincaré, the $L$-index is defined by $I_{L}(X)$ $=\sigma(X \times P) \in L_{n}\left(\pi_{1} X\right)$.

If $X$ is a manifold, this definition has also been suggested by Wall [7, p. 263]. L. Jones points out that Wall's treatment is incomplete in that Lemma 2, p. 264, remains to be proved in the special case $f$ is a simple 
homotopy equivalence. This follows at once from the above, and can probably also be proved using the techniques of [7].

If $X$ is a normal space with Poincare boundary, it is easy to see that $8 \sigma(X)=I_{L}\left(\partial_{P} X\right)$. In particular if $f: X \rightarrow Y$ is a normal map of Poincaré spaces, $8 \sigma(f)=8 \sigma\left(Y_{f}, X \cup Y\right)=I_{L}(X)-I_{L}(Y)$. The 8 in this formula is necessary even in the $\pi_{1}=1$ case.

Now we have $I_{L}: \Omega_{n}^{P}(X) \rightarrow L_{n}\left(\pi_{1} X\right)$, with $I_{L} \circ \partial_{P}=8$. Thus the sequence in 3.1 splits $\otimes Z\left[\frac{1}{2}\right]$. The behavior at 2 when $\pi_{1} X=1$ has also been completely analyzed [5].

Further, if $Y$ is normal without boundary, $f: Y \rightarrow X$, then $8 \sigma(Y, f)$ $=I_{L}(\varnothing)=0 \in L_{n}\left(\pi_{1} X\right)$. Thus $\sigma(8 Y)=0$. Since $M S G \rightarrow K(Z)$ splits $H_{*}(X ; M S G) \rightarrow H_{*}(X ; Z)$ is onto, and the obstruction having exponent 8 implies $\Omega_{*}^{P}(X) \rightarrow H_{*}(X ; \boldsymbol{Z})$ is onto $8 H_{*}(X ; \boldsymbol{Z})$. Since an odd multiple of any class is representable by a manifold, we get

3.3. (Steenrod representability.) $\Omega_{*}^{P}(X) \rightarrow H_{*}(X ; \boldsymbol{Z})$ is onto, ${ }^{*} \neq 3$.

When $\pi_{1} X=1$, this is due to Levitt.

Next there is a natural projection of any group to a free abelian group by abelianizing and dividing out torsion. The image of $I_{L}(X)$ in $L_{n}\left(\pi_{1} X\right)$ $\rightarrow L_{n}\left(Z^{k}\right) /$ torsion is Novikov's multisignature; 2.2. and the splitting theorem for $L$ groups shows it can be obtained as indexes of Poincaré subspaces of a Poincaré space bordant to $X$.

3.4. (Multisignature.) The Novikov multisignature is defined for Poincaré spaces and is an invariant of Poincaré bordisms preserving the projection to $Z^{k}$.

In particular it is a homotopy invariant of smooth manifolds, a conjecture of Novikov. The homotopy invariance was first proved by Hsiang and Shaneson. Up to some powers of 2, G. Lustig has also proved 3.4., and A. S. Mischenko also has a version.

Lastly, we give a product theorem for surgery obstructions. Using the definition of $L_{n}(G)$ as the bordism group of $(n+1)$-dimensional normal spaces with Poincare boundary, there is a pairing by cartesian product $\times: \Omega_{m}^{P}(X) \times L_{n}(G) \rightarrow L_{n+m}\left(\pi_{1} X \times G\right)$. Composing with $\partial_{P}$ gives a pairing $\pi: L_{m}\left(\pi_{1} X\right) \times L_{n}(G) \rightarrow L_{n+m}\left(\pi_{1} X \times G\right) ; \pi=\times \circ\left(\partial_{P} \times 1\right)$.

3.5. (8 Product theorem.) If $X^{m}$ Poincaré, $Y \in L_{m}(G)$, then $8 \sigma(X \times Y)$ $=I_{L}(X) \pi \sigma(Y)$ in $L_{m+n}\left(\pi_{1} X \times G\right)$.

There is also a version for $\boldsymbol{Z} / p$ Poincaré spaces, etc., so for odd primes this gives a fairly complete result. For $\pi_{1} X=1$ the usual (multiplication by the index) theorem can be recovered from this one for $Z / p$ Poincaré spaces, modulo 2-torsion.

There are also applications of this theory to bundle theories which will be considered separately [5]. 


\section{BIBLIOGRAPHY}

1. W. Browder, Surgery on simply-connected manifolds (to appear).

2. L. Jones, Patch spaces (to appear).

3. N. Levitt, On the structure of Poincaré duality spaces, Topology 7 (1968), 369-388. MR 40 \# 2089.

4. - Generalized Thom spectra and transversality for spherical fibrations, Bull. Amer. Math Soc. 76 (1970), 727-731. MR 41 \#2689.

5. F. Quinn, $B_{\left(\mathrm{TOP}_{n}\right)} \sim$ and the surgery obstruction. II (to appear).

6. C. T. C. Wall, Poincaré complexes. I, Ann. of Math. (2) 86 (1967), 213-245. MR 36 \#880.

7. - Surgery of compact manifolds, Academic Press, New York, 1971.

School of Mathematics, Institute for Advanced Study, Princeton, New Jersey 08540 